\title{
RELATO INTEGRADO E DESEMPENHO FINANCEIRO DAS EMPRESAS LISTADAS NA B3
}

\section{Integrated reporting and financial performance of listed companies on B3}

Helenice Souza Gonçalves

E-mail: helenycesg@gmail.com

MestraemCiências Contábeis peloProgramadePós-graduaçãoemCiênciasContábeisdaUniversidadeFederal de Pernambuco; Bacharel em Ciências Contábeis pela Universidade Federal da Bahia. https://orcid.org/0000-0003-3257-2663

Luiz Carlos Marques dos Anjos

E-mail: luizcmanjos@gmail.com

Doutorem Ciências Contábeis pelaUniversidade de Brasília; Mestreem Ciências Contábeis pela Universidade FederaldePernambuco;ProfessorAdjuntonoDepartamentode Ciências ContábeisdaUniversidadeFederalde Pernambuco. Endereço para contato: Avenida Professor Moraes Rego, 1235, Cidade Universitária, 50670-901, Recife, Pernambuco, Brasil. https://orcid.org/0000-0002-7375-540X

Maurício Assuero de Lima Freitas E-mail: massuero@ig.com.br Doutorem EconomiapelaUniversidadeFederaldePernambuco;MestreemEconomiapelaUniversidadeFederal dePernambuco;ProfessorAdjuntonoDepartamentodeCiências ContábeiseAtuariaisdaUniversidadeFederalde

Pernambuco. https://orcid.org/0000-0002-0437-3571

Artigo recebido em 4 de dezembro de 2018. Aceito em 28 de junho de 2019. 


\section{Resumo}

O Relato Integrado tem como proposta a criação de valor para todos os seus stakeholders, organizado em seis capitais: financeiro, manufaturado, natural, intelectual, humano, social e de relacionamento. Esta pesquisa analisou se as empresas listadas na Brasil, Bolsa, Balcão (B3), pertencentes aos setores de bens industriais, materiais básicos, consumo não cíclico e utilidade pública, têm seu desempenho financeiro influenciado pela publicação do Relato Integrado. Por meio da análise de Dados em Painel, com estimação pelos Efeitos Fixos e Efeitos Aleatórios, apurou-se que não há influência da RSC sobre o desempenho financeiro nas empresas que publicam o documento em estudo; esse resultado foi apurado tanto na análise conjunta das empresas quanto na análise por setor de atividade.

Palavras-chave: Relato Integrado. Responsabilidade Social Corporativa. Desempenho financeiro.

\section{Abstract}

The Integrated Report proposes to create value for all its stakeholders, organized in six capitals: financial, manufactured, natural, intellectual, human, social and relationship. This research analyzed whether the companies listed in Brazilian Stock Exchange (B3), belonging to the sectors of industrial goods, basic materials, non-cyclic consumption and public utility, have their financial performance influenced by the publication of the Integrated Report. Through the Panel Data analysis, with estimation of the Fixed Effects and Random Effects it was found that there is no influence of the CSR on the financial performance in the companies that publish the document under study, this result was verified both in the joint analysis of the companies, and in the analysis by sector.

Keywords: Integrated Reporting. Corporate Social Responsibility. Financial performance.

\section{INTRODUÇÃO}

A apresentação de ações de Responsabilidade Social Corporativa (RSC) é uma maneira de obter a legitimidade organizacional e de conseguir a permissão da sociedade para manter as atividades operacionais da empresa. Para conquistar a legitimidade, as companhias precisam divulgar as suas ações para que seu público de interesse tome conhecimento delas, e, uma das formas de propagar essas informações é por meio de relatórios empresariais.

Os relatórios de RSC apresentam informações não financeiras e financeiras das organizações. Nesses documentos são apresentados dados como gastos para a proteção ambiental, filantropia, bem-estar dos empregados e benefícios para a sociedade. A divulgação desses tipos de informações pode influenciar o valor da empresa (Dhaliwal, Li, Tsang, \& Yang, 2014).

Ao longo dos anos surgiram normas para a criação de RSC, como as elaboradas pelo Global Reporting Initiative (GRI), e em 2010 iniciaram-se as discussões do Relato Integrado (RI), um documento com o objetivo de apresentar informações das organizações (financeiras e não financeiras) para diversos públicos e dessa forma gerar valor, tanto para a organização 
quanto para os stakeholders. Para a elaboração de sua Estrutura Conceitual, empresas de diversos países, inclusive do Brasil, participaram de um Projeto-Piloto, no qual as companhias começaram a publicar seus relatórios nas diretrizes sugeridas pelo Integrated Reporting Council (IIRC) e compartilhavam suas experiências na construção do relatório.

O RI propõe a criação de valor baseado em seis capitais: financeiro, manufaturado, natural, intelectual, humano, social e de relacionamento. Com esses capitais são fornecidas informações sobre a empresa tanto para o público interno quanto para o externo.

Desde a sua criação, o Relato Integrado tem ganhado destaque no ambiente corporativo, visto a quantidade de empresas que têm aderido a ele de forma espontânea e também o aumento das regulamentações sobre o assunto (Zhou, Simnett, \& Green, 2017). No Brasil, os relatórios sociais corporativos são voluntários, contudo, a Brasil, Bolsa, Balcão tem incentivado que as empresas listadas publiquem ou o Relatório de Sustentabilidade ou o Relato Integrado.

Dentro do contexto apresentado, questiona-se: qual a relação da publicação do Relato Integrado e o desempenho financeiro das empresas listadas na B3? Para responder a essa pergunta este artigo teve como objetivo analisar a relação do $\mathrm{RI}$ e o desempenho financeiro das companhias. A fim de atingir o objetivo proposto, utilizou-se a regressão de Dados em Painel com a estimação realizada com Efeito Fixo e Efeito Aleatório para a investigação da influência da RSC no desempenho financeiro das empresas listadas na B3.

O desempenho financeiro está contido no Capital Financeiro, e tem sido estudado por diversos autores (Churet \& Eccles, 2014; López, Garcia, \& Rodriguez, 2007; Testa \& D’Amato, 2017)we analyse the relation between CSR and certain accounting indi-cators and examine whether there exist significant dif-ferences in performance indicators between European firms that have adopted CSR and others that have not. The effects of compliance with the requirements of CSR were determined on the basis of firms included in the Dow Jones Sustainability Index (DJSI quando se discute o impacto de RSC nas finanças empresariais. Além de que, sem recursos financeiros, as organizações não conseguem investir em ações socioambientais (Carroll, 1991).

Por o RI ser um documento recente, as empresas ainda estão em processo de adequação (Alves, Kassai, Lucas, \& Ferreira, 2017), dessa forma, esta pesquisa contribui para entender a relação entre o desempenho financeiro e o Relato Integrado no curto prazo das empresas listadas na Bolsa brasileira.

A divisão deste trabalho consiste no referencial teórico, na segunda seção; a metodologia, na terceira seção, que contém os procedimentos utilizados durante a realização da pesquisa; a apresentação do resultado, na quarta seção, em que são evidenciados os principais achados; e as considerações finais, na quinta seção, com as limitações do estudo e sugestões de pesquisa. 


\section{REFERENCIAL TEÓRICO}

\subsection{TEORIA DA LEGITIMIDADE}

Existe uma espécie de "contrato social" (Deegan, Rankin, \& Tobin, 2002; O'Donovan, 2002; Samkin \& Schneider, 2010) entre a organização e a sociedade na qual ela atua; por meio dele a empresa conquista o direito de exercer suas atividades no local onde está instalada. Esse contrato é obtido por meio da legitimidade organizacional, e para alcançá-la a empresa precisa evidenciar que suas atividades convergem com os interesses da sociedade (Deegan et al., 2002; Milne \& Patten, 2002).

A legitimidade é adquirida com o equilíbrio entre os valores da comunidade da qual a empresa faz parte e a sua forma de produção (Dowling \& Pfeffer, 1975). Para encontrar esse meio-termo as organizações estão dispostas a alterar sua maneira de trabalhar para se manterem legítimas perante o olhar da sociedade (Deegan, 2002).

Para se tornar legítimo são necessários três pontos: adaptar suas atividades produtivas para o entendimento atual de legitimidade, influenciar a percepção da sociedade sobre legitimidade por meio de informações da empresa e publicar informes sobre a atividade da organização que vão ao encontro dos valores locais (Dowling \& Pfeffer, 1975).

Uma vez conquistada a legitimidade, a organização precisa continuar o diálogo com a sociedade, pois os valores mudam com o tempo e o que era aceito no passado pode não ser tolerado no futuro (Boulouta \& Pitelis, 2014; Dowling \& Pfeffer, 1975; O'Donovan, 2002; Suchman, 1995). A legitimidade pode ser obtida mediante três tipos de estratégia: pragmática, moral e cognitiva (Suchman, 1995). Por intermédio dessas técnicas as companhias selecionam o ambiente no qual vão atuar e se adaptam a ele ou o manipulam. Segundo Suchman, não existe uma estratégia superior a outra e as empresas podem utilizá-las de forma simultânea.

A pragmática é a adaptação da organização ao público de interesse, a moral está relacionada com os valores e na cognitiva as atividades empresariais são aceitas porque são consideradas essenciais para o cotidiano da sociedade (Suchman, 1995). Com o objetivo de alcançar a legitimidade, as empresas proferem discursos de sustentabilidade em relatórios corporativos, sendo o Relato Integrado um dos relatórios que tem sido utilizado nos últimos anos (Zappettini \& Unerman, 2016). Contudo, se os dados contidos no RI forem divergentes das informações propagadas por outros meios de comunicação, a organização pode perder sua legitimidade perante a sociedade (Casonato, Farneti, \& Dumay, 2018). 


\subsection{RELATO INTEGRADO: GERAÇÃO DE VALOR}

Para atender a diferentes stakeholders, as informações de RSC são agrupadas em quatro dimensões: econômica, legal, ética e filantrópica. A dimensão econômica encontrase na base da pirâmide porque em primeiro lugar a empresa precisa ser lucrativa para dar prosseguimento às suas atividades, mas além do retorno financeiro a organização tem que cumprir a lei, que é a dimensão legal (Carroll, 1991). A dimensão ética relaciona-se com as atitudes da empresa que devem evitar comportamentos que não são aprovados pelos seus públicos de interesse, dos empregados aos consumidores, e, por fim, a filantrópica, situação em que a organização contribui financeiramente para o bem-estar da comunidade (Carroll, 1991).

Sprinkle e Maines (2010) apresentam uma série de motivações para que as empresas realizem ações de RSC. Uma delas é que tais ações contribuem para atrair e manter bons profissionais, outra causa é que a empresa consegue fidelizar os clientes e, dessa forma, aumentar a sua presença no mercado; as preocupações ambientais podem contribuir para a diminuição dos custos de produção.

As empresas se utilizam de ações de Responsabilidade Social Corporativa para obter a sua legitimidade organizacional, e para que a sociedade tenha conhecimento de suas atitudes elas divulgam as informações nos mais diversos documentos e um deles é o Relato Integrado.

As discussões iniciais do RI começaram em 2010 quando foi criado o International Integrated Reporting Council, uma iniciativa do Príncipe de Gales e do Global Reporting Initiative (GRI). Entre julho de 2010 e 2013, empresas, inclusive brasileiras, participaram de um Projeto Piloto com o objetivo de criar um framework para o Relato Integrado.

O Relato Integrado é um desdobramento do Relatório de Sustentabilidade. No Relatório de Sustentabilidade a RSC é apresentada em três dimensões: econômica, social e ambiental. Itens como custo operacional, crescimento das receitas e investimentos são reportados na dimensão econômica (Yadava \& Sinha, 2016). Aspectos como condição de trabalho, remuneração e diversidade de gênero dos profissionais da empresa, segurança e saúde dos trabalhadores são registrados na dimensão social (Yadava \& Sinha, 2016). Na dimensão ambiental são evidenciadas informações como consumo de energia e água e liberação de gases de efeito estufa durante o processo produtivo (Yadava \& Sinha, 2016).

A publicação do RI permite uma visão integrada das empresas por meio de seis capitais: financeiro, manufaturado, natural, intelectual, humano, social e de relacionamento (Baboukardos \& Rimmel, 2016). Capital financeiro é o montante de recursos à disposição da empresa para a fabricação de produtos ou prestação de serviços; o capital manufaturado corresponde a bens que permitem a atividade econômica da organização, como prédios e equipamentos. Os recursos ambientais (renováveis e não renováveis) que possibilitam 
- funcionamento da empresa são considerados capital natural (International Integrated Reporting Council [IIRC], 2013).

A propriedade intelectual e o "capital organizacional" são itens do capital intelectual e estão alicerçados em conhecimento como direitos autorais e protocolos. O capital humano refere-se às pessoas e está pautado na experiência dos funcionários e nos incentivos oferecidos a estes para inovar. A interação e o cuidado entre a empresa e a comunidade fazem parte do capital social de relacionamento, este preocupa-se com a qualidade de vida dos indivíduos (IIRC, 2013).

O mercado de capitais tem valorizado o Relato Integrado, pois este contribui para uma melhor qualidade das informações empresariais e com a publicação desse documento há uma redução da assimetria informacional, o que permite uma melhor acurácia da previsão de analistas (Zhou et al., 2017). Isso leva os investidores a aceitarem um menor retorno financeiro em prol de informações mais confiáveis (Zhou et al., 2017). O RI colabora para a legitimidade em situações em que há uma vulnerabilidade das práticas empresariais e em que o ambiente no qual as organizações estão inseridas são instáveis (Baboukardos \& Rimmel, 2016).

O RI é um documento que abrange dois mundos distintos: o socioambiental e o empresarial (Baboukardos \& Rimmel, 2016; Bommel, 2014), sendo essa interação de informações diferenciadas que permite que o Relato Integrado proporcione valor para os seus diversos públicos de interesse. Esse documento não foi criado para assumir a posição dos demais relatórios, mas para unir as informações existentes neles (Alves et al., 2017).

A publicação de informações econômicas e socioambientais em um único documento, como o RI, permite que as empresas obtenham muitos benefícios, como a manutenção de acionistas e a atração de novos, além de outros stakeholders (Zappettini \& Unerman, 2016). Esses autores relatam ainda que o RI pode ser utilizado como um mecanismo de Relações Públicas, além de ser um relatório que fornece informações para as áreas jurídica e financeira das organizações.

\subsection{ESTUDOS ANTERIORES}

A literatura sobre a relação entre RSC e desempenho financeiro apresenta resultados divergentes. Um aumento do desempenho financeiro ocasionado pela RSC foi identificado por Lee e Yeo (2016), Marti, Rovira-Val, e Drescher (2015), enquanto que López et al. (2007) e Magnanelli e Izzo (2017) identificaram relação inversa, uma redução do desempenho financeiro motivado pela RSC, enquanto que Schreck (2011), Surroca, Tribó, e Waddock (2010) observaram que não há acréscimo ou diminuição do desempenho financeiro das empresas que apresentam ações de RSC. 
Aupperle, Carroll, e Hatfield (1985) não identificaram influência entre RSC (econômico, legal, ético e discricionariedade) e desempenho financeiro, contudo, evidenciaram na pesquisa que as empresas orientadas para resultados econômicos têm poucas preocupações com os elementos relacionados à RSC.

López et al. (2007) compararam empresas listadas na Dow Jones Global Index (DJGI) e na Dow Jones Sustainability Index (DSII). Eles identificaram que no curto prazo a RSC implica a redução do desempenho financeiro da empresa. Essa influência negativa é explicada pela necessidade de gastos iniciais para atender aos requisitos para ser considerada uma organização socialmente responsável. A proxy utilizada para a RSC foi a empresa estar ou não listada na DSJI.

Na pesquisa realizada por Baird, Geylani, e Roberts (2012) detectou-se que os valores do desempenho financeiro são aumentados em razão da RSC para alguns setores econômicos, como óleo e gás, embalagens e têxteis e reduzidos para conglomerados industriais. Entre as variáveis analisadas, pode-se citar retorno sobre capital investido, vendas, e endividamento, e para mensurar a RSC, utilizou-se a variável DIM que representa algumas dimensões, como governança, direitos humanos e diversidade.

Vantagem competitiva, reputação e satisfação do cliente foram as proxies utilizadas por Saeidi, Sofian, Saeidi, Saeidi, e Saaeidi (2015) para avaliar a relação entre a RSC e o desempenho financeiro empresarial. Os autores identificaram que somente a vantagem competitiva e a reputação são variáveis que medeiam positivamente a conexão entre a RSC e o desempenho financeiro da organização.

As dimensões da RSC podem ser analisadas individualmente ou como um conjunto. Miras-Rodríguez, Carrasco-Gallego, e Escobar-Pérez (2015) analisaram as dimensões de forma individualizada e perceberam que o olhar ambiental da RSC não tem influência sobre o desempenho financeiro, contudo, outras dimensões (comunidade, diversidade, governança corporativa e responsabilidade do produto) contribuem para o acréscimo do ROA.

Com relação à dimensão ambiental, foi identificado por Testa e D'Amato (2017) o mesmo resultado de Miras-Rodríguez et al. (2015), de que as ações ambientais não afetam o desempenho financeiro, contudo, verificou-se que um bom desempenho financeiro contribui para o investimento de ações ambientalmente responsáveis.

As companhias com alta complexidade organizacional são mais beneficiadas com a publicação de relatórios de RSC no momento da avaliação empresarial do que as com menor complexidade organizacional (Lee \& Yeo, 2016). Percebeu-se que a qualidade do RI reduz a assimetria informacional entre as pessoas que estão dentro da organização e as que são externas à empresa, o que contribui para baixos custos de financiamento. Esse resultado diverge do trabalho de Magnanelli e lzzo (2017), que identificaram que quanto maiores os recursos para ações de RSC, maior o custo da dívida. 
Na pesquisa conduzida por Magnanelli e Izzo (2017) foram analisadas 332 empresas de diferentes setores econômicos e de diversos países. Os autores evidenciaram que os bancos (financiadores das empresas analisadas) percebem os investimentos em RSC como um desperdício de recursos e, por esse motivo, o custo da dívida tem seus valores majorados.

Relacionando, especificamente, a relação entre o desempenho financeiro e o Relato Integrado, Churet e Eccles (2014) concluíram que o último não altera o primeiro no curto prazo, entretanto, analisando os setores individualmente, observou-se que nos de saúde e tecnologia da informação o desempenho financeiro tem seus valores incrementados em razão da RSC.

O estudo da influência de oito elementos do RI sobre o desempenho financeiro de empresas da Malásia foi realizado por Wen e Heong (2017), que concluíram que quatro deles (governança, modelo do negócio, risco e oportunidades e divulgação do desempenho) têm um impacto positivo significativo no desempenho financeiro.

Ao analisar a qualidade dos Relatos Integrados, Barth, Cahan, Chen, e Venter (2018) detectaram que essa característica contribui para uma melhor liquidez para as empresas. Eles identificaram também que a qualidade dos Relatos Integrados permite uma tomada de decisão mais prudente, o que melhora a eficiência dos investimentos. Carp, Păvăloaia, Afrăsinei, e Georgescu (2019) pesquisaram a relação entre o RI e o crescimento das empresas da Romênia e encontraram que há fraca relação entre essas variáveis.

Percebe-se que não há um consenso sobre qual o impacto da publicação do RI para a geração de valor para os acionistas, tendo como medidas indicadores de desempenho financeiro e por indicadores do mercado de capitais.

\section{METODOLOGIA}

\subsection{EMPRESAS ANALISADAS}

As empresas analisadas (Tabela 1) são as que participaram do projeto-piloto do Relato Integrado e as que pertencem ao mesmo segmento. Foram excluídas as empresas do setor financeiro e as com dados incompletos. A classificação do setor seguiu a mesma divisão utilizada pela Brasil, Bolsa, Balcão. Analisou-se o período entre 2013 e 2017.

A distinção de quais empresas possuem Relatório de Sustentabilidade, Relatório Integrado ou nenhum dos dois foi realizada por meio do informativo da B3 intitulado Empresas Listadas: Relatório de Sustentabilidade ou Integrado. Por esta publicação da B3, não é possível saber qual o tipo de relatório de RSC que é veiculado, portanto, visitaram- 
se os sites das empresas que informaram utilizar ou o Relatório de Sustentabilidade ou o Relato Integrado para identificar quais publicam este último.

Algumas empresas como as de energia e de concessionária de rodovias individualmente não emitem o Relato Integrado, mas o grupo ao qual pertencem faz a publicação do RI; nessa situação foi considerado que a organização divulga tal documento. Assim como Alves et al. (2017), percebeu-se que poucas empresas adotam o nome Relato/Relatório Integrado, as organizações reportam que seguem as diretrizes do RI no decorrer do documento.

Tabela 1

Empresas analisadas por setor de atividade

\begin{tabular}{ll}
\hline Bens industriais & 43 \\
Consumo não cíclico & 17 \\
Materiais básicos & 17 \\
Utilidade pública & 58 \\
\hline Total & 135 \\
\hline
\end{tabular}

As empresas listadas no setor de Bens Industriais são as que exercem suas atividades no comércio, construção e engenharia, máquinas e equipamentos, material de transporte, serviços e transportes. No consumo não cíclico, agropecuária, alimentos processados, bebidas, comércio e distribuição, produtos diversos e produtos de uso pessoal e de limpeza. As empresas de embalagens, madeira e papel, materiais diversos, mineração e químicos fazem parte do setor de materiais básicos, enquanto as empresas de água e saneamento, energia elétrica e gás pertencem ao setor de utilidade pública.

\subsection{DEFINIÇÃO DAS VARIÁVEIS}

O modelo utilizado neste artigo é adaptado do estudo de López et al. (2007):

$$
\text { LAIR }_{i t}=\beta_{1}+\beta_{2} \text { RSC }_{i t-1}+\beta_{3} \text { TAM }_{i t}+\beta_{4} \text { ENDIVIDAMENTO }_{i t}+\beta_{5} \text { SETOR }_{i}+\varepsilon_{i t}
$$

Para uma maior robustez, analisaram-se os setores individualmente:

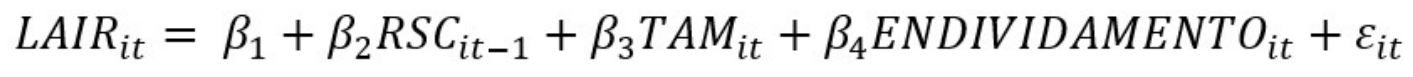

Na Tabela 2 é apresentado o significado de cada variável utilizada nesta pesquisa. A variável RSC está no ano anterior para capturar o efeito da Responsabilidade Social 
Corporativa, que é refletida ao longo do tempo (Tebini, M'Zali, Lang, \& Perez-Gladish, 2016). $\mathrm{Na}$ Tabela 3 também é apresentado o sinal esperado para cada variável. As variáveis que são representadas por valores monetários foram transformadas em logaritmos, antes desse procedimento elas foram elevadas ao quadrado, pois algumas possuíam valores negativos.

Tabela 2

Variáveis do modelo

\begin{tabular}{ll}
\hline LAIR & $\begin{array}{l}\text { Lucro/prejuízo antes do imposto de renda-variação para o tempo } \dagger \text { em relação } \\
\text { ao período-base }\end{array}$ \\
\hline RSC & Dummy-l se publica relato integrado, o caso contrário \\
\hline TAM. (controle) & Total do ativo-variação para o tempo + em relação ao período-base \\
\hline $\begin{array}{l}\text { ENDIVIDAMENTO } \\
\text { (controle) }\end{array}$ & Débito sobre ativo-variação para o tempo $\dagger$ em relação ao período-base \\
\hline SETOR (controle) & Setor da atividade econômica \\
\hline
\end{tabular}

Tabela 3

Sinais esperados

\begin{tabular}{lcl}
\hline \multicolumn{1}{c}{ Variável } & Sinal esperado & \multicolumn{1}{c}{ Autor(es) } \\
\hline RSC & + & Lee e Yeo (2016) e Marti et al. (2015) \\
RSC & - & Magnanelli e lzzo (2017) e López et al. (2007) \\
TAM. & - & Schreck (2011) \\
ENDIVIDAMENTO & - & Schreck (2011) \\
SETOR & - & López et al. (2007) \\
\hline
\end{tabular}

As informações foram coletadas na base de dados disponibilizados pelo projeto GetDFPData; este projeto é mantido pelo Professor Marcelo Perlin. Através do sítio eletrônico do projeto ou de um pacote específico para o programa $R$, é possível coletar as informações de forma gratuita das empresas listadas na B3. Para a obtenção dos resultados, utilizou-se o software Eviews 10 Student Version Lite.

O teste de Hausman (Tabela 4) indicou a utilização de efeitos fixos para o modelo principal, bens industriais, e de efeitos aleatórios para consumo não cíclico, materiais básicos e utilidade pública.

Tabela 4

Teste de Hausman

\begin{tabular}{cl}
\hline Principal & 0.0041 \\
\hline Bens industriais & 0.0067 \\
\hline Consumo não cíclico & 0.0067 \\
\hline Materiais básicos & 0.4648 \\
\hline Utilidade pública & 0.0700 \\
\hline
\end{tabular}


A normalidade dos dados foi verificada por meio do teste de Jarque-Bera, a autocorrelação pelo teste de Durbin-Watson e a heterocedasticidade pelo teste de White. A variável Setor (Equação 1) foi excluída em razão da multicolinearidade.

\section{RESULTADOS}

Com base estatística descritiva (Tabela 5 ) pode-se afirmar que os valores médios para Ativo, Receita e LAIR são maiores para as empresas que emitem o RI, enquanto a variável Risco assume os valores mais altos para as organizações do setor consumo não cíclico e materias básicos que não publicam o Relato Integrado. As maiores médias para LAIR das empresas que divulgam o RI corroboram os resultados de Marti et al. (2015), que identificaram que companhias com ações de RSC têm um desempenho financeiro superior do que as outras empresas.

Tabela 5

Estatística descritiva (valores em log)

\begin{tabular}{|c|c|c|c|c|c|c|c|c|}
\hline & \multicolumn{4}{|c|}{ Com RI } & \multicolumn{4}{|c|}{ Sem RI } \\
\hline & Mín. & Méd. & Máx. & $\begin{array}{l}\text { Desv. } \\
\text { Pad. }\end{array}$ & Mín. & Méd. & Máx. & $\begin{array}{l}\text { Desv. } \\
\text { Pad. }\end{array}$ \\
\hline \multicolumn{9}{|c|}{ Ativo } \\
\hline Bens industriais & 12.286 & 12.853 & 13.791 & 0.5676 & 8.3721 & 12.523 & 17.238 & 1.7218 \\
\hline consumo não cíclico & 13.302 & 14.184 & 15.361 & 0.8089 & 9.2733 & 13.252 & 16.176 & 1.9338 \\
\hline Materiais básicos & 13.909 & 14.893 & 16.079 & 0.5705 & 9.7114 & 12.725 & 16.295 & 1.5435 \\
\hline Utilidade pública & 13.225 & 14.314 & 15.251 & 0.5347 & 8.5329 & 13.119 & 16.476 & 1.4070 \\
\hline \multicolumn{9}{|c|}{ Endividamento } \\
\hline Bens industriais & 1.8089 & 1.8567 & 1.8857 & 0.0218 & 1.2658 & 1.8252 & 2.1785 & 0.0943 \\
\hline Consumo não cíclico & 1.6916 & 1.8347 & 1.9019 & 0.0797 & 1.6649 & 1.8559 & 2.0246 & 0.0695 \\
\hline Materiais básicos & 1.7913 & 1.8321 & 1.8543 & 0.0160 & 1.6794 & 1.8339 & 2.1851 & 0.0809 \\
\hline Utilidade pública & 1.7436 & 1.8554 & 1.8941 & 0.0285 & 1.4099 & 1.8248 & 2.1570 & 0.0771 \\
\hline \multicolumn{9}{|c|}{ LAIR } \\
\hline Bens industriais & 10.685 & 11.350 & 12.047 & 0.4797 & 0.9542 & 10.005 & 14.974 & 1.8649 \\
\hline Consumo não cíclico & 11.003 & 11.867 & 12.816 & 0.4530 & 5.5253 & 10.583 & 14.436 & 1.9384 \\
\hline Materiais básicos & 8.5029 & 11.410 & 12.967 & 1.4434 & 5.9663 & 10.065 & 14.412 & 1.6004 \\
\hline Utilidade pública & 5.4049 & 11.072 & 13.053 & 1.3766 & 2.3522 & 10.431 & 14.307 & 1.7748 \\
\hline
\end{tabular}

Como pode ser observado na matriz de correlação (Tabela 6), as variáveis possuem uma relação fraca entre elas, com exceção de Tamanho e LAIR, que apresentaram forte relação positiva em todas as análises. Exceto por Risco e RSC para os setores de consumo não cíclico e materiais básicos, todas as demais correlações apresentaram relação positiva, indicando que as variáveis se movem no mesmo sentido. 
Tabela 6

Matriz de correlação

\begin{tabular}{|c|c|c|c|c|c|c|c|}
\hline & LAIR & RSC & TAM. & ENDIVID & BENS & CONSUMO & MAT. \\
\hline \multicolumn{8}{|c|}{ Painel A: Modelo principal } \\
\hline LAIR & 1.000000 & & & & & & \\
\hline RSC & 0.183334 & 1.000000 & & & & & \\
\hline TAM. & 0.751100 & 0.253965 & 1.000000 & & & & \\
\hline ENDIVID. & 0.259459 & 0.096830 & 0.252697 & 1.000000 & & & \\
\hline BENS. IND. & -0.10497 & -0.10033 & -0.213011 & 0.007636 & 1.000000 & & \\
\hline CONSUMO & 0.07436 & -0.009194 & 0.082300 & 0.099543 & -0.25944 & 1.000000 & \\
\hline MATERIAIS & 0.0062734 & 0.058140 & 0.040735 & 0.033421 & -0.257248 & -0.1450014 & 1.000000 \\
\hline \multicolumn{8}{|c|}{ Painel B: Bens industriais } \\
\hline LAIR & 1.000000 & & & & & & \\
\hline RSC & 0.303180 & 1.000000 & & & & & \\
\hline TAM. & 0.765227 & 0.052874 & 1.000000 & & & & \\
\hline ENDIVID. & 0.190508 & 0.091669 & 0.043597 & 1.000000 & & & \\
\hline \multicolumn{8}{|c|}{ Painel C: Consumo não cíclico } \\
\hline LAIR & 1.000000 & & & & & & \\
\hline RSC & 0.209485 & 1.000000 & & & & & \\
\hline TAM. & 0.726040 & 0.142686 & 1.000000 & & & & \\
\hline ENDIVID. & 0.120787 & -0.104029 & 0.184339 & 1.000000 & & & \\
\hline \multicolumn{8}{|c|}{ Painel D: Materiais básicos } \\
\hline LAIR & 1.000000 & & & & & & \\
\hline RSC & 0.292990 & 1.000000 & & & & & \\
\hline TAM. & 0.647949 & 0.487115 & 1.000000 & & & & \\
\hline ENDIVID. & 0.319896 & -0.048184 & -0.003327 & 1.000000 & & & \\
\hline \multicolumn{8}{|c|}{ Painel E: Utilidade pública } \\
\hline LAIR & 1.000000 & & & & & & \\
\hline RSC & 0.121363 & 1.000000 & & & & & \\
\hline TAM. & 0.743969 & 0.318911 & 1.000000 & & & & \\
\hline ENDIVID. & 0.365841 & 0.201569 & 0.558408 & 1.000000 & & & \\
\hline
\end{tabular}

A variável RSC não apresentou relevância estatística nem para o modelo principal (Tabela 7) nem para a análise individual por setor (Tabela 8). As variáveis que apresentaram significância estatística para o modelo principal (Tabela 7) foram Tamanho e Risco, enquanto que na análise por setor de atividade (Tabela 8), em materiais básicos as variáveis que influenciam o desempenho financeiro são tamanho e risco, e para utilidade pública a variável estatisticamente relevante foi Tamanho. Para bens industriais nenhuma das variáveis apresentou significância estatística. 
Tabela 7

Resultado do modelo principal

\begin{tabular}{|c|c|}
\hline Variável & Coeficiente \\
\hline Constante & $\begin{array}{c}-3.905148 \\
(3.330838)\end{array}$ \\
\hline RSC & $\begin{array}{c}-0.362658 \\
(0.299690)\end{array}$ \\
\hline TAM. & $\begin{array}{c}0.436963^{* * *} \\
(0.156080)\end{array}$ \\
\hline ENDIVID. & $\begin{array}{c}4.710243^{* * *} \\
(1.297965)\end{array}$ \\
\hline $\mathrm{R} 2$ & 0.796188 \\
\hline Estatística $\mathrm{F}$ & 11.37476 \\
\hline Probabilidade (Estatística F) & 0.000000 \\
\hline
\end{tabular}

Nota. Entre parênteses o erro padrão, ${ }^{* * *}$ significativo a $1 \%$. As variá-

veis de setor foram excluídas em razão da multicolinearidade exata.

Apesar de indícios da influência da publicação do RI com o desempenho financeiro observados nas Tabelas 6 e 8 , os resultados corroboram os achados de Churet e Eccles (2014), que relataram que o Relato Integrado não altera o desempenho financeiro no curto prazo e com outras pesquisas (Schreck, 2011; Surroca et al., 2010), as quais investigaram outros tipos de ações de RSC.

Tabela 8

Resultado por setor de atividade

\begin{tabular}{ccccc}
\hline Variáveis & Bens industriais & Consumo não cíclico & Materiais básicos & Utilidade pública \\
\hline \multirow{2}{*}{ Constante } & $24.09253^{* * *}$ & 5.573332 & $-13.47457^{* * *}$ & -1.978837 \\
& $(10.40668)$ & $(4.359628)$ & $(4.260501)$ & $(2.362392)$ \\
RSC & -0.510570 & 0.149273 & -0.053826 & -0.482548 \\
& $(1.063053)$ & $(0.451772)$ & $(0.460988)$ & $(0.277363)$ \\
TAM. & -0.885867 & $0.865928^{* * *}$ & $0.696228^{* * *}$ & $0.935216^{* * *}$ \\
& $(0.553959)$ & $(0.865928)$ & $(0.114338)$ & $(0.094711)$ \\
ENDIVID. & -1.525887 & -3.494349 & $7.978720 * *$ & 0.059361 \\
R2 & $(2.350281)$ & $(2.381701)$ & $(2.188918)$ & $(1.487770)$ \\
Estatística F & 0.819627 & 0.561607 & 0.487638 & 0.362614 \\
Probabilidade (Es- & 12.70269 & 27.32929 & 19.98661 & 42.85783 \\
tatística F) & 0.000000 & 0.000000 & 0.000000 & 0.000000 \\
\hline
\end{tabular}

Nota. Entre parênteses o erro padrão, ${ }^{* * *}$ significativo a $1 \%$.

As empresas estão em processo de adaptação ao Relato Integrado (Alves et al., 2017) e os resultados positivos relacionados à RSC não são percebidos no curto prazo (Tebini et al., 2016), portanto, pode ser que no futuro as empresas listadas na B3 que emitem o RI tenham seu resultado influenciado pela divulgação desse documento. Contudo, cabe ressaltar que nem sempre a influência de relatórios socioambientais sobre o desempenho financeiro ocorre de forma positiva (Baboukardos \& Rimmel, 2016). 
Nesse contexto, a manutenção da divulgação do RI pelas empresas, apesar de não haver um acréscimo no desempenho financeiro, pode ser explicada porque essa publicação permite a obtenção de legitimidade organizacional (Zappettini \& Unerman, 2016), o que confere a oportunidade do prosseguimento das atividades organizacionais.

\section{CONCLUSÃO}

O RI surge com a proposta de gerar valor para todas as partes interessadas. Nele as informações sobre a empresa são organizadas em seis capitais: financeiro, manufaturado, natural, intelectual, humano, social e de relacionamento.

Os resultados indicam que as empresas listadas na B3 não têm o seu desempenho financeiro alterado pela publicação do Relato Integrado. $O$ retorno financeiro é apenas uma das motivações para que se emita esse relatório; pode ser que as empresas estejam publicando para sinalizar ao seu público de interesse como estão conciliando as atividades econômicas com as questões socioambientais, conquistando, dessa forma, legitimidade organizacional.

Assim como divergências entre as práticas empresariais e as informações do RI contribuem para que as organizações percam a legitimidade perante a sociedade, pode ser que as partes interessadas ainda não tenham conhecimento sobre o Relato Integrado, que é um relatório recente. Isso pode contribuir para que os benefícios esperados com a publicação do documento não sejam alcançados.

Uma limitação da pesquisa se concentra no fato de que faz pouco tempo que as empresas adotaram o Relato Integrado, e os efeitos da adoção de RSC no resultado financeiro não são percebidos no curto prazo, portanto, uma sugestão de pesquisa é que essa análise seja realizada posteriormente contemplando um período maior de tempo.

Futuras pesquisas poderiam comparar o comportamento do desempenho financeiro e da Responsabilidade Social Corporativa apenas de empresas que são engajadas com a RSC, mas que emitem relatórios distintos, a exemplo do Relatório de Sustentabilidade e do Relato Integrado.

\section{REFERÊNCIAS}

Alves, N. J. F., Kassai, J. R., Lucas, E. C., \& Ferreira, H. M. G. (2017). Relato Integrado e o formato da informação financeira para evidenciar a criação de valor das empresas do Programa Piloto. Revista Evidenciação Contábil \& Finanças, 5(3), 99-122. Recuperado de https://doi.org/10.18405/recfin20170306 
Aupperle, K. E., Carroll, A. B., \& Hatfield, J. D. (1985). An empirical examination of the relationship between corporate social responsability and profitability. The Academy of Man agement Journal, 28(2), 446-463.

Baboukardos, D., \& Rimmel, G. (2016). Value relevance of accounting information under an integrated reporting approach: A research note. Jornal of Accounting and Public Policy, 35(4), 437-452. Recuperado de https://doi.org/10.1016/j.jaccpubpol.2016.04.004

Baird, P. L., Geylani, P. C., \& Roberts, J. A. (2012). Corporate social and financial performance re-examined: Industry effects in a linear mixed model analysis. Journal of Business Ethics, 109(3), 367-388. Recuperado de https://doi.org/10.1007/s10551-011-1135-z

Barth, M. E., Cahan, S. F., Chen, L., \& Venter, E. R. (2018). The economic consequences associated with integrated report quality: Capital market and real effects. Accounting, Organizations and Society, 62, 43-64. Recuperado de https://doi.org/10.1016/j.aos.2017.08.005

Bommel, K. van. (2014). Towards a legitimate compromise? An exploration of Integrated Reporting in the Netherlands. Accounting, Auditing \& Accountability Journal, 27(7), 1157 1189. Recuperado de https://doi.org/10.1108/AAAJ-04-2013-1309

Boulouta, I., \& Pitelis, C. N. (2014). Who needs CSR? The impact of corporate social responsibility on national competitiveness. Journal of Business Ethics, 119(3), 349-364. Recuperado de https://doi.org/10.1007/s10551-013-1633-2

Carp, M., Păvăloaia, L., Afrăsinei, M.-B., \& Georgescu, I. E. (2019). Is sustainability reporting a business strategy for firm's growth? Empirical study on the Romanian capital market. Sustainability, 11(658), 1-21. Recuperado de https://doi.org/10.3390/sul1030658

Carroll, A. B. (1991). The pyramid of corporate social responsibiiity: Toward the moral management of organizational stakeholders. Business Horizons, 34, 39-48. Recuperado de https://doi.org/10.1177/0312896211432941

Casonato, F., Farneti, F., \& Dumay, J. (2018). Social capital and integrated reporting: Losing legitimacy when reporting talk is not supported by actions. Journal of Intellectual Capital, 20(1), 144-164. Recuperado de https://doi.org/10.1108/JIC-08-2018-0132

Churet, C., \& Eccles, R. G. (2014). Integrated Reporting, quality of management, and financial performance. Journal of Applied Corporate Finance, 26(1), 56-64. Recuperado de https://doi.org/10.1007/978-1-4614-9173-6

Deegan, C. (2002). Introduction: The legitimising effect of social and environmental disclousures - a theoretical foundation. Accounting, Auditing \& Accountability Journal, 15(3), 282-311. Recuperado de https://doi.org/10.1108/09513570210435852 
Deegan, C., Rankin, M., \& Tobin, J. (2002). An examination of the corporate social and environmental disclosures of BHP from 1983-1997: A test of legitimacy theory. Accounting, Auditing \& Accountability Journal, 15(3), 312-343. Recuperado de https://doi. org/10.1108/09513570210435861

Dhaliwal, D., Li, O. Z., Tsang, A., \& Yang, Y. G. (2014). Corporate social responsibility disclosure and the cost of equity capital: The roles of stakeholder orientation and financial transparency. Journal of Accounting and Public Policy, 33(4), 328-355. Recuperado de https://doi.org/10.1016/j.jaccpubpol.2014.04.006

Dowling, J., \& Pfeffer, J. (1975). Organizational legitimacy: Social values and organizational behavior. The Pacific Sociological Review, 18(1), 122-136. Recuperado de https://doi. org/10.2307/1388226

International Integrated Reporting Council. (2013). Business and Investors Explore the Sustainability Perspective of Integrated Reporting.

Lee, K. W., \& Yeo, G. H. H. (2016). The association between integrated reporting and firm valuation. Review of Quantitative Finance and Accounting, 47(4), 1221-1250. Recuperado de https://doi.org/10.1007/s11156-015-0536-y

López, M. V., Garcia, A., \& Rodriguez, L. (2007). Sustainable development and corporate performance: A study based on the Dow Jones Sustainability Index. Journal of Business Ethics, 75(3), 285-300. Recuperado de https://doi.org/10.1007/s10551-006-9253-8

Magnanelli, B. S., \& Izzo, M. F. (2017). Corporate social performance and cost of debt: The relationship. Social Responsibility Journal, 13(2), 250-265. Recuperado de https://doi. org/10.1108/SRJ-06-2016-0103

Marti, C. P., Rovira-Val, M. R., \& Drescher, L. G. J. (2015). Are firms that contribute to sustainable development better financially? Corporate Social Responsibility and Environmental Management, 22(5), 305-319. Recuperado de https://doi.org/10.1002/csr.1347

Milne, M. J., \& Patten, D. M. (2002). Securing organizational legitimacy. Accounting, Auditing \& Accountability Journal, 15(3), 372-405. Recuperado de https://doi. org/10.1108/09513570210435889

Miras-Rodríguez, M. del M., Carrasco-Gallego, A., \& Escobar-Pérez, B. (2015). Has the CSR engagement of electrical companies had an effect on their performance? A closer look at the environment. Business Strategy and the Environment, 24(8), 819-835. Recuperado de https://doi.org/10.1002/bse.1848 
O'Donovan, G. (2002). Environmental disclosures in the annual report: Extending the applicability and predictive power of legitimacy theory. Accounting, Auditing \& Accountability Journal, 15(3), 344-371. Retrieved from https://doi.org/10.1108/09513570210435870

Perlin, M., Kirch, G., \& Vancin, D. (2018, February 22). Accessing Financial Reports and Corporate Events with GetDFPdata. Retrieved from SSRN:https://ssrn.com/abstract $=3128252$

Saeidi, S. P., Sofian, S., Saeidi, P., Saeidi, S. P., \& Saaeidi, S. A. (2015). How does corporate social responsibility contribute to firm financial performance? The mediating role of competitive advantage, reputation, and customer satisfaction. Journal of Business Research, 68(2), 341-350. Retrieved from https://doi.org/10.1016/j.jbusres.2014.06.024

Samkin, G., \& Schneider, A. (2010). Accountability, narrative reporting and legitimation The case of a New Zealand public benefit entity. Accounting, Auditing \& Accountability Journal, 23(2), 256-289. Retrieved from https://doi.org/10.1108/09513571011023219

Schreck, P. (2011). Reviewing the business case for Corporate Social Responsibility: New evidence and analysis. Journal of Business Ethics, 103(2), 167-188. Retrieved from https://doi. org/10.1007/s10551-011-0867-0

Sprinkle, G. B., \& Maines, L. A. (2010). The benefits and costs of corporate social responsibility. Business Horizons, 53(5), 445-453. Retrieved from https://doi.org/10.1016/j.bushor.2010.05.006

Suchman, M. C. (1995). Managing Legitimacy: Strategic and Institucional Approaches. Academy of Management Review, 2O(3), 571-610. Retrieved from https://doi.org/10.5465/ AMR.1995.9508080331

Surroca, J., Tribó, J. A., \& Waddock, S. (2010). Corporate Responsability and financial performance: The role of intangible resources. Strategic Management Journal, 31(5), 463 490. Retrieved from https://doi.org/10.1002/smj

Tebini, H., M'Zali, B., Lang, P., \& Perez-Gladish, B. (2016). The economic impact of environmentally responsible practices. Corporate Social Responsibility and Environmental Management, 23(5), 333-344. Retrieved from https://doi.org/10.1002/csr.1383

Testa, M., \& D'Amato, A. (2017). Corporate environmental responsibility and financial performance: Does bidirectional causality work? Social Responsibility Journal, 13(2), 221-234. Retrieved from https://doi.org/10.1108/SRJ-02-2016-0031

Wen, L. P., \& Heong, A. Y. K. (2017). Integrated Reporting and financial performance: Evidence from Malaysia. Management \& Accounting Review, 16(2). 
Yadava, R. N., \& Sinha, B. (2016). Scoring sustainability reports using GRI 2011 Guidelines for assessing environmental, economic, and social dimensions of leading public and private Indian companies. Journal of Business Ethics, 138(3), 549-558. Retrieved from https://doi.org/10.1007/s10551-015-2597-1

Zappettini, F., \& Unerman, J. (2016). "Mixing" and "Bending": the recontextualisation of discourses of sustainability in integrated reporting. Discourse \& Communication, 10(5), 521542. Retrieved from https://doi.org/10.1177/1750481316659175

Zhou, S., Simnett, R., \& Green, W. (2017). Does Integrated Reporting matter to the capital market? Abacus, 53(1), 94-132. Retrieved from https://doi.org/10.1111/abac.12104

\section{Como citar este artigo:}

\section{ABNT}

GONÇALVES, Helenice Souza; MARQUES DOS ANJOS, Luiz Carlos; LIMA FREITAS, Maurício Assuero de. Relato integrado e desempenho financeiro das empresas listadas na B3. RACE, Revista de Administração, Contabilidade e Economia, Joaçaba: Ed. Unoesc, v. 18, n. 2, p. 345-362, maio/ago. 2019. Disponível em: http://editora.unoesc.edu.br/index.php/ race. Acesso em: dia/mês/ano.

\section{APA}

Gonçalves, H. S., Marques dos Anjos, L. C., \& Lima Freitas, M. A. de (2019). Relato integrado e desempenho financeiro das empresas listadas na B3. RACE, Revista de Administração, Contabilidade e Economia, 18(2), 345-362. Recuperado de http://editora.unoesc. edu.br/index.php/race 